\title{
Asymptotic behavior in a model with Yukawa interaction from Schwinger-Dyson equations
}

\author{
V.E. Rochev ${ }^{1}$ \\ Institute for High Energy Physics, 142280 Protvino, Russia
}

\begin{abstract}
A system of Schwinger-Dyson equations for pseudoscalar four-dimensional Yukawa model in the two-particle approximation is investigated. The simplest iterative solution of the system corresponds to the mean-field approximation (or, equivalently, to the leading order of $1 / N$-expansion) and includes a non-physical Landau pole in deep-Euclidean region for the pseudoscalar propagator $\Delta$. It is argued, however, that a full solution may be free from non-physical singularities and has the self-consistent asymptotic behavior $p_{e}^{2} \Delta \simeq C \log ^{-4 / 5} \frac{p_{e}^{2}}{M^{2}}$. An approximate solution confirms the positivity of $C$ and the absence of Landau pole.

PACS number: 11.10.Jj.
\end{abstract}

\section{Introduction}

A definition of asymptotic behavior at large momenta for the strictly renormalized four-dimensional models of quantum field theory (QFT) up to now is an unsolved problem. The unique exception is the theory with non-Abelian gauge interaction, which is asymptotically free in the framework of renormalization-group-improved perturbation theory. A solution of the problem of asymptotic behavior for other models requires going out the framework of the coupling-constant perturbative expansion. The first attempt to define the asymptotic behavior in QFT was made by Landau and coworkers in the 1950s. This investigation was based on the approximate solution of Dyson equations with the summation of leading logarithms, and the result for quantum electrodynamics was as follows: the photon propagator included the non-physical singularity in Euclidean region of momenta [1]. Then similar singularities were indicated in the model with Yukawa interaction [2]. Such singularities in the Euclidean region violate general principles of QFT and are a serious problem for these models. Further development has demonstrated that these non-physical singularities arise practically inevitably in the framework of any known non-perturbative methods: at the renormalization-group summation, in the frameworks of $1 / N$-expansion and mean-field expansion, etc. ${ }^{2}$

A widespread opinion is formulated as a triviality of the quantum field models that is not asymptotically free in the sense of the improved coupling-constant perturbative expansion. There is a rigorous theorem [4] that the four-dimensional scalar field theory with $\phi^{4}$ interaction on the lattice does not have an interacting

\footnotetext{
${ }^{1}$ E-mail address: rochev@ihep.ru

${ }^{2}$ See reviews [3] for the historical survey and further references.
} 
continuum theory as its limit for zero lattice spacing, i.e. the theory is trivial. However, this argument is not fully conclusive due to an uncertainty of the continuous limit in this model [5].

In spite of the serious evidence for the triviality of lattice scalar theory, the situation with triviality today is as vague as before, and recent papers in this topic maintain incompatible statements. While mainstream works confirm the triviality scenario, Suslov in a series of works(see [6] and references therein) argues the nontrivial behavior for $\phi^{4}$-theory and quantum electrodynamics in the strong-coupling region. In any case, a study of the triviality problem requires a non-perturbative tool.

In this paper we investigate a new non-perturbative approximation for a model with Yukawa interaction - two-particle approximation, or 2PA. This approximation was proposed for scalar fields in [7], and it is the first non-trivial step of a sequence of general $n$-particle approximations, which tends to the exact infinite system of Schwinger-Dyson equations (SDEs) at $n \rightarrow \infty$.

The structure of the paper is as follows: in section 2, the necessary notations and definitions are given; SDEs for the generating functional of Green functions are introduced in the formalism of a bilocal fermion source ${ }^{3}$. We consider using of the bilocal source as a convenient choice of the functional variable. In particular, this variable is very convenient for the construction of the mean-field (MF) expansion, which is presented in section 2. The existence of the Landau pole in the boson propagator of the leading approximation of the MF expansion is also demonstrated in this section.

In section 3, a general construction of the approximation scheme for the system of SDEs is given. The renormalization of the system of equations is made, iterative solutions and some supplement simplifications are discussed.

In section 4 , the asymptotic solution of the system at large Euclidean momenta is presented and the asymptotic behavior of the propagators at large momenta is discussed. The boson propagator in this model possesses self-consistent behavior. Conclusions are presented in section 5 .

\section{Schwinger-Dyson equations, mean-field approx- imation and Landau pole}

Consider the theory of a Dirac fermion field $\psi$ interacting with a pseudoscalar boson field $\phi$ in a four-dimensional $(1+3)$ space with the Lagrangian

$$
\mathcal{L}=\bar{\psi}(i \hat{\partial}-m) \psi-\frac{1}{2} \phi\left(M^{2}+\partial^{2}\right) \phi+g \bar{\psi} \Gamma \psi \phi
$$

\footnotetext{
${ }^{3} \mathrm{~A}$ formalism of the bilocal source was first elaborated in QFT by Dahmen and JonaLasinio [8].
} 
Here $\Gamma=i \gamma^{5}$ and $\hat{\partial}=\gamma^{\mu} \partial_{\mu}$. The renormalizability in all orders of the couplingconstant expansion requires to supplement Lagrangian (1) with an additional term $\lambda \phi^{4}$ which corresponds to the self-interaction of the boson field. This term ensures a renormalization of the boson-boson scattering amplitude. In this work we are not concerned with this amplitude, and the renormalization of approximations considered below does not require including the corresponding counter-term. For this reason we do not include the quartic interaction into consideration. Therefore, we shall consider the restricted Yukawa model neglecting the self-interaction of the boson field, and the obtained results should be treated as the first step to the study of the asymptotic behavior in a realistic model of the boson-fermion interaction. In other words, we shall consider the case $\lambda=0$ only. Also we shall believe $m>0$ and $M^{2}>0$ and, therefore, do not discuss in this work the problem of dynamical mass generation by Yukawa interaction (see [9]).

The generating functional of Green functions can be written as a functional integral

$$
G=\int D(\psi, \bar{\psi}, \phi) \exp i\left[\int d x \mathcal{L}-\int d x d y \bar{\psi}(y) \eta(y, x) \psi(x)+\int d x j(x) \phi(x)\right],
$$

where $j(x)$ is a single boson source and $\eta(x, y)$ is a bilocal fermion source.

The translational invariance of the functional integration measure leads to the functional-differential SDEs for generating functional $G$. In terms of the logarithm $Z=\frac{1}{i} \log G$ these equations are:

$$
\begin{array}{r}
\delta(x-y)+\left(i \hat{\partial}_{x}-m\right) i \frac{\delta Z}{\delta \eta(y, x)}+g \Gamma\left[\frac{\delta^{2} Z}{\delta \eta(y, x) \delta j(x)}+i \frac{\delta Z}{\delta \eta(y, x)} \frac{\delta Z}{\delta j(x)}\right]= \\
=\int d x_{1} \eta\left(x, x_{1}\right) i \frac{\delta Z}{\delta \eta\left(y, x_{1}\right)} \\
\frac{\delta Z}{\delta j(x)}=\int d x_{1}\left[\Delta_{c}\left(x-x_{1}\right) j\left(x_{1}\right)-g \Delta_{c}\left(x-x_{1}\right) \operatorname{tr}\left(\Gamma \frac{\delta Z}{\delta \eta\left(x_{1}, x_{1}\right)}\right)\right] .
\end{array}
$$

Here $\Delta_{c}=\left(M^{2}+\partial^{2}\right)^{-1}$. We define also the fermion propagator

$$
S(x-y)=\left.i \frac{\delta Z}{\delta \eta(y, x)}\right|_{\eta=j=0},
$$

the boson propagator

$$
\Delta(x-y)=\left.\frac{\delta^{2} Z}{\delta j(y) \delta j(x)}\right|_{\eta=j=0}
$$

the two-particle (four-point) fermion function

$$
Z_{2}\left(\begin{array}{cc}
x & y \\
x^{\prime} & y^{\prime}
\end{array}\right)=\left.i \frac{\delta^{2} Z}{\delta \eta\left(y^{\prime}, x^{\prime}\right) \delta \eta(y, x)}\right|_{\eta=j=0}
$$


and the three-point function

$$
G_{3}(z \mid x, y)=\left.i \frac{\delta^{2} Z}{\delta \eta(y, x) \delta j(z)}\right|_{\eta=j=0} .
$$

Differentiations of SDE (4) over $\eta$ and $j$ give us the SDE for the three-point function

$$
G_{3}(z \mid x, y)=-g \int d z_{1} \Delta_{c}\left(z-z_{1}\right) \Gamma Z_{2}\left(\begin{array}{cc}
x & y \\
z_{1} & z_{1}
\end{array}\right)
$$

and the SDE for the boson propagator

$$
\Delta(x-y)=\Delta_{c}(x-y)+i g \int d y_{1} \operatorname{tr}\left[\Gamma G_{3}\left(x \mid y_{1}, y_{1}\right)\right] \Delta_{c}\left(y_{1}-y\right) .
$$

Excluding with the help of SDE (4) a differentiation over $j$ in SDE (3), we obtain at $j=0$ the SDE for the generating functional:

$$
\begin{array}{r}
\delta(x-y)+\left(i \hat{\partial}_{x}-m\right) i \frac{\delta Z}{\delta \eta(y, x)}=\int d x_{1}\left\{i \eta\left(x, x_{1}\right) \frac{\delta Z}{\delta \eta\left(y, x_{1}\right)}+\right. \\
\left.+g^{2} \Delta_{c}\left(x-x_{1}\right) \Gamma\left[i \frac{\delta Z}{\delta \eta(y, x)} \operatorname{tr}\left(\Gamma \frac{\delta Z}{\delta \eta\left(x_{1}, x_{1}\right)}\right)+\frac{\delta}{\delta \eta(y, x)} \operatorname{tr}\left(\Gamma \frac{\delta Z}{\delta \eta\left(x_{1}, x_{1}\right)}\right)\right]\right\}
\end{array}
$$

which contains only the derivatives over the bilocal source $\eta$. Switching off the source $\eta$ in (11), we have the SDE for the fermion propagator

$$
\left(m-i \hat{\partial}_{x}\right) S(x-y)=\delta(x-y)+i g^{2} \int d x_{1} \Delta_{c}\left(x-x_{1}\right) \Gamma Z_{2}\left(\begin{array}{cc}
x & y \\
x_{1} & x_{1}
\end{array}\right) \Gamma
$$

A differentiation of (11) over $\eta$ gives us (with the source being switched off) the SDE for the two-particle fermion function

$$
\begin{array}{r}
\left(m-i \hat{\partial}_{x}\right) Z_{2}\left(\begin{array}{cc}
x & y \\
x^{\prime} & y^{\prime}
\end{array}\right)+\delta\left(x-y^{\prime}\right) S\left(x^{\prime}-y\right)= \\
=i g^{2} \int d x_{1}\left\{(\Gamma S(x-y)) \Delta_{c}\left(x-x_{1}\right) Z_{2}\left(\begin{array}{cc}
x_{1} & x_{1} \\
x^{\prime} & y^{\prime}
\end{array}\right) \Gamma+\Gamma \Delta_{c}\left(x-x_{1}\right) Z_{3}\left(\begin{array}{cc}
x & y \\
x_{1} & x_{1} \\
x^{\prime} & y^{\prime}
\end{array}\right) \Gamma\right\}
\end{array}
$$

Here $Z_{3}=\left.i \frac{\delta^{3} Z}{\delta \eta^{3}}\right|_{\eta=0}$ is the three-particle (six-point) fermion function. The derivation of equation (13) implies that $\operatorname{tr}\left(\gamma^{5} S\right)=0$, i.e. we suppose parity conservation.

To construct the MF expansion, we consider as a leading approximation for equation (11) the equation

$$
\begin{array}{r}
\delta(x-y)+\left(i \hat{\partial}_{x}-m\right) i \frac{\delta Z^{M F}}{\delta \eta(y, x)}= \\
i \int d x_{1}\left\{\eta\left(x, x_{1}\right) \frac{\delta Z^{M F}}{\delta \eta\left(y, x_{1}\right)}+g^{2} \Delta_{c}\left(x-x_{1}\right) \Gamma \frac{\delta Z^{M F}}{\delta \eta(y, x)} \operatorname{tr}\left[\Gamma \frac{\delta Z^{M F}}{\delta \eta\left(x_{1}, x_{1}\right)}\right]\right\}
\end{array}
$$


We call (14) the MF approximation, since this equation gives us the same equations for propagators and the two-particle funcion as the MF expansion for the generating functional (see [7] and references therein).

The MF fermion propagator is

$$
S=S^{c},
$$

where $S^{c}=(m-i \hat{\partial})^{-1}$.

Equation (14) gives the equation for the two-particle function

$$
\begin{array}{r}
Z_{2}\left(\begin{array}{cc}
x & y \\
x^{\prime} & y^{\prime}
\end{array}\right)+S^{c}\left(x-y^{\prime}\right) S^{c}\left(x^{\prime}-y\right)= \\
=i g^{2} \int d x_{1} d x_{2}\left(S^{c}\left(x-x_{1}\right) \Gamma S^{c}\left(x_{1}-y\right)\right) \Delta_{c}\left(x_{1}-x_{2}\right) Z_{2}\left(\begin{array}{cc}
x_{2} & x_{2} \\
x^{\prime} & y^{\prime}
\end{array}\right) \Gamma
\end{array}
$$

whose solution is

$$
\begin{array}{r}
Z_{2}\left(\begin{array}{cc}
x & y \\
x^{\prime} & y^{\prime}
\end{array}\right)=-S^{c}\left(x-y^{\prime}\right) S^{c}\left(x^{\prime}-y\right)+ \\
+\int d x_{1} d x_{2}\left(S^{c}\left(x-x_{1}\right) \Gamma S^{c}\left(x_{1}-y\right)\right) f_{M F}\left(x_{1}-x_{2}\right)\left(S^{c}\left(x^{\prime}-x_{2}\right) \Gamma S^{c}\left(x_{2}-y^{\prime}\right)\right),
\end{array}
$$

where in the momentum space

$$
\frac{1}{f_{M F}\left(p^{2}\right)}=\frac{i}{g^{2}} \Delta_{c}^{-1}\left(p^{2}\right)+L^{c}\left(p^{2}\right),
$$

and

$$
L^{c}\left(p^{2}\right)=\int \frac{d^{4} q}{(2 \pi)^{4}} \operatorname{tr}\left[S^{c}(p+q) \Gamma S^{c}(q) \Gamma\right]
$$

is the single fermion loop.

Taking into account SDEs (9) and (10), we obtain the MF boson propagator

$$
\Delta\left(p^{2}\right)=\frac{i}{g^{2}} f_{M F}\left(p^{2}\right) .
$$

The above formulae contain divergent integrals and should be renormalized.

The simplest method of renormalization of the above equations is the direct application of a regularization procedure in the spirit of Bogolyubov R-operation [10]. The unrenormalized mass operator is

$$
\sigma\left(p^{2}\right)=\Delta^{-1}\left(p^{2}\right)-\Delta_{c}^{-1}\left(p^{2}\right)=-i g^{2} L^{c}\left(p^{2}\right) .
$$

Then the renormalized mass operator is defined as

$$
\sigma_{r}\left(p^{2}\right)=\operatorname{reg} \sigma\left(p^{2}\right)=-i g^{2} L_{r}^{c}\left(p^{2}\right),
$$


where

$$
L_{r}^{c}\left(p^{2}\right)=L^{c}\left(p^{2}\right)-L^{c}(0)-p^{2}\left(L^{c}\right)^{\prime}(0)=\frac{i p^{2}}{8 \pi^{2}} \int_{0}^{1} d z \log \left[1-z(1-z) \frac{p^{2}}{m^{2}}\right] .
$$

is the renormalized fermion loop ( for the easement of the following calculations we choose the normalization point at zero momenta). As a result, the renormalized boson propagator $\Delta_{r}$ is

$$
\Delta_{r}^{-1}\left(p^{2}\right)=M^{2}-p^{2}-i g^{2} L_{r}^{c}\left(p^{2}\right)
$$

We can also renormalize the MF approximation by introducing counter-terms in the Lagrangian. In correspondence with the standard recipe, we consider (1) as the renormalized Lagrangian, where $\psi, \phi, m, M$ and $g$ are now the renormalized fields, masses and coupling, and add counter-terms

$$
\Delta \mathcal{L}=-\frac{z_{\phi}-1}{2} \phi \partial^{2} \phi-\frac{\delta M^{2}}{2} \phi^{2}
$$

which absorb the divergences.

The full Lagrangian $\mathcal{L}_{b}=\mathcal{L}+\Delta \mathcal{L}$ can be written as

$$
\mathcal{L}_{b}=\mathcal{L}+\Delta \mathcal{L}=\bar{\psi}_{b}\left(i \hat{\partial}-m_{b}\right) \psi_{b}-\frac{1}{2} \phi_{b}\left(M_{b}^{2}+\partial^{2}\right) \phi_{b}+g_{b} \bar{\psi}_{b} \Gamma \psi_{b} \phi_{b}
$$

where

$$
\psi_{b}=\psi, \phi_{b}=\sqrt{z_{\phi}} \phi, g_{b}=\frac{g}{\sqrt{z_{\phi}}}, m_{b}=m, M_{b}^{2}=\frac{M^{2}+\delta M^{2}}{z_{\phi}} .
$$

Then all the above calculations are reproduced with Lagrangian (26), and the normalization conditions are imposed on the renormalized propagator $\Delta_{r}$.

The normalization conditions for the propagator $\Delta_{r}\left(p^{2}\right)=z_{\phi}^{-1} \Delta_{b}\left(p^{2}\right)$ are

$$
\Delta_{r}^{-1}(0)=M^{2},\left.\frac{d}{d p^{2}} \Delta_{r}^{-1}\right|_{p^{2}=0}=1 .
$$

These conditions define the mass-renormalization counter-term $\delta M^{2}$ and the fieldrenormalization constant $z_{\phi}$. Then the renormalized boson propagator is defined by equation (24) as above.

As it follows from equation (24), the renormalized boson propagator $\Delta_{r}$ possesses a non-physical singularity (Landau pole) in the Euclidean region $p^{2}<0$ at the point $p_{e}^{2} \equiv-p^{2}=M_{L}^{2}$, where $M_{L}^{2}$ is a solution of the equation

$$
M^{2}+M_{L}^{2}-i g^{2} L_{r}^{c}\left(-M_{L}^{2}\right)=0 .
$$

This equation has a solution at any positive $g^{2}$. As was yet noted in the introduction, the same Landau pole arises in the calculations of the renormalized amplitude by other methods: in the frameworks of $1 / N$-expansion and renormalization-group summation. 


\section{The system of SDEs and two-particle approxi- mation}

The system of fermion SDEs is an infinite set of equations for $n$-particle fermion functions $Z_{n} \equiv i \delta^{n} Z /\left.\delta \eta^{n}\right|_{\eta=0}$. The first SDE is equation (12). The second SDE is equation (13). The $n$th SDE is the $(n-1)$ th derivative of SDE (11) with the source being switched off and includes a set of functions from one-particle fermion function $S$ to $(n+1)$-particle fermion function $Z_{n+1}$.

We call "the $n$-particle approximation of the system of SDEs" the system of $n$ SDEs in which the first $n-1$ equations are exact and the $n$th SDE is truncated by omitting the $(n+1)$-particle function. It is evident that the sequence of such approximations goes to the exact set of SDEs at $n \rightarrow \infty$. The one-particle approximation is simply equation (12) without $Z_{2}$. This approximation has a trivial solution which is a free propagator. The two-particle approximation is a system of equation (12) and equation (13) without $Z_{3}$ :

$$
\begin{array}{r}
\left(m-i \hat{\partial}_{x}\right) Z_{2}\left(\begin{array}{cc}
x & y \\
x^{\prime} & y^{\prime}
\end{array}\right)+\delta\left(x-y^{\prime}\right) S\left(x^{\prime}-y\right)= \\
=i g^{2} \int d x_{1}(\Gamma S(x-y)) \Delta_{c}\left(x-x_{1}\right) Z_{2}\left(\begin{array}{cc}
x_{1} & x_{1} \\
x^{\prime} & y^{\prime}
\end{array}\right) \Gamma
\end{array}
$$

which includes $S$ and two-particle function $Z_{2}$. This nonlinear system will be the object of the present investigation.

The idea of these approximation scheme is very simple and natural. However, the calculations became more and more complicated at each following stage: e.g., the three-particle approximation is a system of three nonlinear equations for the propagator, the two-particle function and the three-particle function.

Another view to the origin of system (12) and (29) is based on a modification of the MF expansion of section 2 with taking into account a particular solution of functional-derivative equation (11). It is easy to see that the SDE (11) has the simple solution

$$
Z_{p}(\eta)=\frac{1}{2 i} Z_{2} \cdot \eta^{2}-i S \cdot \eta
$$

where functions $Z_{2}\left(\begin{array}{cc}x & y \\ x^{\prime} & y^{\prime}\end{array}\right)$ and $S(x-y)$ satisfy the system of equations (12) and (29). To be an exact solution of the functional-derivative equation (11), this system should be supplemented by one more nonlinear equation for $Z_{2}$. The system of three equations for the two functions $S$ and $Z_{2}$ are overfull and seemingly has not physically meaningful solutions. However, the third equation does not play a role for the construction of the modified MF expansion. The construction of this expansion for scalar field theory can be found in work [7]. This construction can be generalized also to fermion fields. At the $n$th step of this expansion, we have a closed system of linear integral equations, and therefore this scheme is much less complicated in the calculational sense in comparison to the above scheme of the 
$n$-particle approximations. Equations (12) and (29) are the basic approximation for this expansion.

Equations (12) and (29) are the system of nonlinear equations for the functions $S$ and $Z_{2}$. In equation (29), the two-particle function $Z_{2}$ can be considered as a functional of $S$, and the "solution" of this equation can be easily found:

$$
\begin{array}{r}
Z_{2}\left(\begin{array}{cc}
x & y \\
x^{\prime} & y^{\prime}
\end{array}\right)=-S^{c}\left(x-y^{\prime}\right) S\left(x^{\prime}-y\right) \\
+\int d x_{1} d x_{2}\left(S^{c}\left(x-x_{1}\right) \Gamma S\left(x_{1}-y\right)\right) f\left(x_{1}-x_{2}\right)\left(S\left(x^{\prime}-x_{2}\right) \Gamma S^{c}\left(x_{2}-y^{\prime}\right)\right)
\end{array}
$$

Here

$$
f(x-y)=-i g^{2} \Delta_{c}(x-y)+i g^{2} \int d x_{1} d x_{2} \Delta_{c}\left(x-x_{1}\right) L\left(x_{1}-x_{2}\right) f\left(x_{2}-y\right)
$$

and $L(x)=\operatorname{tr}\left[S^{c}(x) \Gamma S(-x) \Gamma\right]$ is the fermion loop operator.

In momentum space:

$$
\begin{gathered}
\frac{1}{f\left(p^{2}\right)}=\frac{i}{g^{2}} \Delta_{c}^{-1}\left(p^{2}\right)+L\left(p^{2}\right), \\
L\left(p^{2}\right)=\int \frac{d^{4} q}{(2 \pi)^{4}} \operatorname{tr}\left[S^{c}(p+q) \Gamma S(q) \Gamma\right] .
\end{gathered}
$$

Taking into account equations (31)-(34), (9) and (10), we obtain for the boson propagator $\Delta$ the following equation in momentum space:

$$
\Delta^{-1}\left(p^{2}\right)=M^{2}-p^{2}-i g^{2} L\left(p^{2}\right) .
$$

From equation (12) and equations (31)-(35) we have the equation for the fermion propagator

$$
S^{-1}(p)=m-\hat{p}+i g^{2} K(p),
$$

where

$$
K(p)=\int \frac{d^{4} q}{(2 \pi)^{4}} \Gamma S^{c}(p-q) \Gamma \Delta(q) .
$$

The system of equations (34)-(37) is the system of unrenormalized SDEs in the 2PA.

The renormalization of equations (35) and (36) can be performed in correspondence with the general recipe of section 2 .

If we define the unrenormalized mass operators as

$$
\left\{\begin{array}{l}
\Sigma(p)=S^{-1}(p)-S_{c}^{-1}(p)=i g^{2} K(p) \\
\sigma\left(p^{2}\right)=\Delta^{-1}\left(p^{2}\right)-\Delta_{c}^{-1}\left(p^{2}\right)=-i g^{2} L\left(p^{2}\right)
\end{array}\right.
$$


then the renormalized mass operators are

$$
\left\{\begin{array}{l}
\Sigma_{r}(p) \equiv \operatorname{reg} \Sigma(p)=i g^{2}\left(K(p)-K(0)-\left.\hat{p} \frac{\partial K}{\partial \hat{p}}\right|_{p=0}\right) \\
\sigma_{r}\left(p^{2}\right) \equiv \operatorname{reg} \sigma\left(p^{2}\right)=-i g^{2}\left(L\left(p^{2}\right)-L(0)-p^{2} L^{\prime}(0)\right)
\end{array},\right.
$$

and the system of renormalized equations for propagators are

$$
\left\{\begin{array}{l}
S_{r}^{-1}(p)=m-\hat{p}+i g^{2} K_{r}(p) \\
\Delta_{r}^{-1}\left(p^{2}\right)=M^{2}-p^{2}-i g^{2} L_{r}\left(p^{2}\right)
\end{array}\right.
$$

where

$$
\begin{gathered}
K_{r}(p)=K(p)-K(0)-\left.\hat{p} \frac{\partial K}{\partial \hat{p}}\right|_{p=0}, \\
L_{r}\left(p^{2}\right)=L\left(p^{2}\right)-L(0)-p^{2} L^{\prime}(0) .
\end{gathered}
$$

The renormalization with counter-terms can be performed by introducing the counter-term Lagrangian

$$
\Delta \mathcal{L}=\left(z_{\psi}-1\right) \bar{\psi} i \hat{\partial} \psi-\delta m \bar{\psi} \psi-\frac{z_{\phi}-1}{2} \phi \partial^{2} \phi-\frac{\delta M^{2}}{2} \phi^{2}
$$

and full Lagrangian (26). The bare quantities in (26) are now

$$
\psi_{b}=\sqrt{z_{\psi}} \psi, \phi_{b}=\sqrt{z_{\phi}} \phi, g_{b}=\frac{g}{z_{\psi} \sqrt{z_{\phi}}}, m_{b}=\frac{m+\delta m}{z_{\psi}}, M_{b}^{2}=\frac{M^{2}+\delta M^{2}}{z_{\phi}} .
$$

The bare mass operators $\Sigma_{b}$ and $\sigma_{b}$ in the 2PA are given by formulae (38) with substitutions $\Sigma \rightarrow \Sigma_{b}, \sigma \rightarrow \sigma_{b}, g \rightarrow g_{b}, m \rightarrow m_{b}, M^{2} \rightarrow M_{b}^{2}$, etc. Normalization conditions (28) for the propagator $\Delta_{r}\left(p^{2}\right)$ and for the fermion propagator

$$
S_{r}^{-1}(p=0)=m,\left.\frac{\partial S_{r}^{-1}(p)}{\partial \hat{p}}\right|_{p=0}=-1
$$

define the counter-terms, and the system of renormalized equations will be system (40) again.

Note that an iteration of equation for $\Delta_{r}$ in (40) with $S^{(0)}=S^{c}$ leads to the MF propagator (24). So the MF approximation and the equivalent leading-order $1 / N$-expansion are contained in the $2 \mathrm{PA}$ as the first iteration.

Equations (40) are the system of nonlinear integral equations for the propagators. The most interesting problem is to look for the asymptotic behavior of the solution of system (40) at large Euclidean momenta. In the large-momenta region, an essential technical simplification is possible, namely, one can replace in integrals (34) and (37) the function $S_{c}$ by a massless function $-1 / \hat{p}$ :

$$
\int \frac{d^{4} q}{(2 \pi)^{4}} F(q) S_{c}(p-q) \Longrightarrow-\int \frac{d^{4} q}{(2 \pi)^{4}} \frac{F(q)}{\hat{p}-\hat{q}} .
$$


Then it is possible to use the well-known formula

$$
\int \frac{d^{4} q_{e}}{(2 \pi)^{4}} \frac{f\left(q_{e}^{2}\right)}{(p-q)_{e}^{2}}=\frac{1}{16 \pi^{2}}\left[\frac{1}{p_{e}^{2}} \int_{0}^{p_{e}^{2}} f\left(q_{e}^{2}\right) q_{e}^{2} d q_{e}^{2}+\int_{p_{e}^{2}}^{\infty} f\left(q_{e}^{2}\right) d q_{e}^{2}\right]
$$

This massless-integration approximation (46) is quite usual in investigations in the deep-Euclidean region, though rigorous arguments for its validity can be done for the asymptotically-free models only [5]. In the general case, this approximation should be considered as a plausible conjecture, which needs further investigations. Formula (47) highly enables the calculations and, as a major point, permits us to go from integral equations to differential ones (see below).

Equations (40) in the massless-integration approximation in the Euclidean region are

$$
\left\{\begin{array}{l}
a\left(p_{e}^{2}\right)=1-\frac{g^{2}}{32 \pi^{2}\left(p_{e}^{2}\right)^{2}} \int_{0}^{p_{e}^{2}} d q_{e}^{2}\left(p_{e}^{2}-q_{e}^{2}\right)^{2} \Delta_{r}\left(q_{e}^{2}\right) \\
\Delta_{r}^{-1}\left(p_{e}^{2}\right)=M^{2}+p_{e}^{2}-\frac{g^{2}}{8 \pi^{2} p_{e}^{2}} \int_{0}^{p_{e}^{2}} d q_{e}^{2}\left(p_{e}^{2}-q_{e}^{2}\right)^{2} \frac{a\left(q_{e}^{2}\right)}{m^{2}+q_{e}^{2} a^{2}}
\end{array}\right.
$$

Here $a$ is defined by $S_{r}^{-1}$ with formula

$$
S_{r}^{-1}=b-a \hat{p}
$$

and $b=m$ in the massless-integration approximation. Euclidean normalization conditions are

$$
\Delta_{r}^{-1}(0)=M^{2},\left(\Delta_{r}^{-1}\right)^{\prime}(0)=1, a(0)=1 .
$$

Introducing the dimensionless quantities

$$
t=\frac{p_{e}^{2}}{M^{2}}, \mu^{2}=\frac{m^{2}}{M^{2}}, \quad h(t)=\frac{1}{p_{e}^{2} \Delta_{r}},
$$

system (48) can be written as follows

$$
\left\{\begin{array}{l}
a(t)=1-\frac{g^{2}}{32 \pi^{2}} \int_{0}^{t}\left(1-\frac{t_{1}}{t}\right)^{2} \frac{d t_{1}}{t_{1} h\left(t_{1}\right)} \\
h(t)=1+\frac{1}{t}-\frac{g^{2}}{8 \pi^{2}} \int_{0}^{t}\left(1-\frac{t_{1}}{t}\right)^{2} \frac{d t_{1} a\left(t_{1}\right)}{\mu^{2}+t_{1} a^{2}\left(t_{1}\right)}
\end{array}\right.
$$

Let us discuss the iterative solutions of system (50). Due to the nonlinearity of equations an essential moment is a way of iterations. Consider two schemes of iterations. The first scheme is a choice $a^{(0)}=1$ as a leading order, and then the calculation of $h^{(1)}$ from the second equation with $a=a^{(0)}$. As was pointed above, this scheme corresponds to the MF approximation and leads to the Landau pole in the boson propagator $\Delta_{r}$ at point $t_{L}$. At this point $h^{(1)}\left(t_{L}\right)=0$, and the following calculation of $a^{(1)}$ from the first equation is possible only at $t<t_{L}$. For this reason the subsequent calculations in the framework of this scheme became problematical in the region of large $t$. 
Another iterative scheme is a choice of the leading order as $h_{0}=1+\frac{1}{t}$, and the following calculation of $a_{1}$ from the first equation with $h=h_{0}$ :

$$
a_{1}=-\frac{g^{2}}{32 \pi^{2}}\left(1+\frac{1}{t}\right)^{2} \log (1+t)+1+\frac{3 g^{2}}{64 \pi^{2}}+\frac{g^{2}}{32 \pi^{2} t} .
$$

Likewise $h^{(1)}$ in the first scheme, function $a_{1}(t)$ equals to zero at some point $t_{0}$, but consequences of this fact are quite different. Since $\mu^{2}+t a_{1}^{2}>0$ at any $t>0,{ }^{4}$ then $h_{1}$ can be calculated for any $t$, where at $t \rightarrow \infty$

$$
h_{1} \simeq 4 \log \log t>0,
$$

i.e., $h_{1}$ has a self-consistent asymptotic behavior. Certainly, a finite number of iteration cannot define the actual asymptotic behavior, but the given considerations demonstrate the obvious preference of the second scheme in comparison with the usual MF approximation. This preference consists in the absence of the Landau pole in the fermion propagator, which is defined by $a_{1}$ of equation (51) at $\mu^{2}>0$. This circumstance is essentially used for the construction of an approximate solution in the following section.

\section{Asymptotic behavior}

System (50) (multiplied to $t^{2}$ ) after threefold differentiations is reduced to the system of differential equations

$$
\left\{\begin{array}{l}
\frac{d^{3}}{d t^{3}}\left(t^{2} a\right)=-\frac{g^{2}}{16 \pi^{2}} \frac{1}{t h} \\
\frac{d^{3}}{d t^{3}}\left(t^{2} h\right)=-\frac{g^{2}}{4 \pi^{2}} \frac{a}{\mu^{2}+a^{2} t}
\end{array}\right.
$$

At $t \rightarrow 0$ system of integral equations (50) gives us:

$$
a(t)=1-\frac{g^{2}}{96 \pi^{2}} t+\frac{g^{2}}{384 \pi^{2}} t^{2}+O\left(t^{3}\right)
$$

and

$$
h(t)=\frac{1}{t}+1-\frac{g^{2}}{24 \pi^{2} \mu^{2}} t+O\left(t^{2}\right)
$$

These formulae give us boundary conditions for system (53) at the point $t=0$.

At large $t$, system (53) has the asymptotic solution ${ }^{5}$ :

$$
a \simeq \mathcal{A} \log ^{1 / 5} t, h \simeq \mathcal{B} \log ^{4 / 5} t, \mathcal{A B}=-\frac{5 g^{2}}{32 \pi^{2}} .
$$

\footnotetext{
${ }^{4} \mathrm{~A}$ case $\mu^{2}=0$ needs a special consideration and is not discussed here.

${ }^{5}$ Note that system (53) has exact solution $a=C t^{-1 / 2}, h=\frac{g^{2} t^{1 / 2}}{6 \pi^{2} C}, C^{2}=-\frac{5}{9} \mu^{2}$. This imaginary solution is not, of course, a solution of integral equations (50).
} 
Differential equations (53) do not fix the signs of coefficients $\mathcal{A}$ and $\mathcal{B}$. These signs have the principal meaning and define the physical situation, described by 2PA. If $\mathcal{A}>0(\mathcal{B}<0)$, then function $h(t)$ applies to zero at some point. This case corresponds to the presence of Landau-type singularity in the boson propagator, i.e., the situation is similar to the above physically unsatisfactory MF approximation. Oppositely, if $\mathcal{A}<0(\mathcal{B}>0)$, then the situation corresponds to self-consistent asymptotic behavior of the boson propagator. In this case, function $a(t)$ has a zero at some point, but it does not lead to a Landau pole in the propagator for massive fermions.

System (53) of nonlinear differential equations is rather difficult for detailed analytical investigation, and below we make some approximations and natural simplified suppositions, which will enable the construction of an approximate solution and the definition of the coefficients $\mathcal{A}$ and $\mathcal{B}$.

Firstly note, that function $v=a h$ has quite definite asymptotic behavior at $t \rightarrow \infty$ :

$$
v=a h \simeq-\frac{5 g^{2}}{32 \pi^{2}} \log t
$$

Since $v \simeq \frac{1}{t}$ at $t \rightarrow 0$, this function changes the sign in some point $t_{0}$ and $v\left(t_{0}\right)=0$. We shall suppose the uniqueness of this point. Consider the behavior of the solution near the point $t_{0}$. After the change of variable $x=\log t$, the first equation of system (53) can be written as

$$
a^{\prime \prime \prime}+3 a^{\prime \prime}+2 a^{\prime}=-\frac{g^{2}}{16 \pi^{2}} \frac{1}{h}=-\frac{g^{2}}{16 \pi^{2}} \frac{a}{v} .
$$

Here $a^{\prime} \equiv \frac{d a}{d x}$. Suppose in a vicinity of zero point $x_{0}=\log t_{0}$ :

$$
v \approx v^{\prime}\left(x_{0}\right)\left(x-x_{0}\right)
$$

Note that supposition (59) is fulfilled for iterative solution (51), which will be used in the construction of the approximate solution (see below).

Then, going to the variable $z=x-x_{0}=\log \frac{t}{t_{0}}$, we obtain for $a$ the linear differential equation

$$
a^{\prime \prime \prime}+3 a^{\prime \prime}+2 a^{\prime}=k \frac{a}{z}
$$

where $k=-\frac{g^{2}}{16 \pi^{2}} \frac{1}{v^{\prime}\left(x_{0}\right)}$. Since $v$ decreases, $k>0$. With the substitution

$$
a=e^{-z} u
$$

and by changing the variable $\xi=\frac{z^{2}}{4}$, we obtain for $u$ the Meijer equation [11]

$$
\left[\xi\left(\xi \frac{d}{d \xi}+\frac{k}{2}\right)-\left(\xi \frac{d}{d \xi}-1\right)\left(\xi \frac{d}{d \xi}-\frac{1}{2}\right) \xi \frac{d}{d \xi}\right] u=0
$$

whose solution is

$$
u=C_{1} u_{1}+C_{2} u_{2}+C_{3} u_{3},
$$


where

$$
\begin{array}{r}
u_{1}=G_{13}^{11}\left(-\frac{z^{2}}{4} \mid \begin{array}{c}
1-k / 2 \\
0 ; \frac{1}{2}, 1
\end{array}\right), u_{2}=i G_{13}^{11}\left(-\frac{z^{2}}{4} \mid \begin{array}{c}
1-k / 2 \\
\frac{1}{2} ; 0,1
\end{array}\right), \\
u_{3}=G_{13}^{21}\left(\frac{z^{2}}{4} \mid \begin{array}{c}
1-k / 2 \\
0,1 ; \frac{1}{2}
\end{array}\right) .
\end{array}
$$

At $z \rightarrow 0$ we have $u_{1} \sim z^{2}, u_{2} \sim z, u_{3} \sim z^{0}$. Consequently, if $C_{3} \neq 0$, function $a$ does not change sign at point $x_{0}$, and $\mathcal{A}>0$. If $C_{3}=0, C_{2} \neq 0$, function $a$ changes sign, and $\mathcal{A}<0$. This case corresponds to the above-mentioned situation of selfconsistent asymptotic behavior. The case $C_{3}=C_{2}=0$ corresponds to touching for $a$ and a pole singularity for $h$.

A definition of coefficients $C_{i}$ needs some boundary conditions. These boundary conditions should be connected with boundary conditions at $t=0$ and can be defined on the basis of some approximate solution in the region of small $t$. The linearized version type of equation (60) is tightly connected with the asymptotic behavior at large $t$ and apparently cannot be applied in the region of small $t$. For this reason, in the pre-asymptotic region we shall use iterative solution (51), which satisfies supposition (59) and boundary conditions (54). In other words, we believe $a=a_{1}$ at $t \leq t_{0}\left(x \leq x_{0}\right)$. In the region of large $t$, we use asymptotic formula (57) for $v$ and believe $v=-\frac{5 g^{2}}{32 \pi^{2}}\left(x-x_{0}\right)$ at $t \geq t_{0}\left(x \geq x_{0}\right)$. Correspondingly, we have for $a$ at large $t$ equation (60), whose solution is given by equations (61)-(63) with $k=2 / 5$.

As the boundary conditions we shall use the conditions of "a smooth join" at point $x=x_{0}(z=0)$ :

$$
a(z=0)=a\left(x_{0}\right)=a_{1}\left(x_{0}\right)=0, \quad a_{z}^{\prime}(0)=a_{1}^{\prime}\left(x_{0}\right), \quad a_{z z}^{\prime \prime}(0)=a_{1}^{\prime \prime}\left(x_{0}\right) .
$$

From these boundary conditions, we define

$$
C_{1}=\frac{\sqrt{\pi}}{\Gamma(1.2)}\left(a_{1}^{\prime \prime}\left(x_{0}\right)+2 a_{1}^{\prime}\left(x_{0}\right)\right), \quad C_{2}=\frac{\pi}{\Gamma(0.7)} a_{1}^{\prime}\left(x_{0}\right), \quad C_{3}=0 .
$$

Here $\Gamma(\kappa)$ is the Euler gamma-function. Simple calculation shows that both $C_{1}$ and $C_{2}$ are negative. Asymptotic expansions of Meijer functions (see [11]) give us the behavior of solution at $z \rightarrow+\infty$ :

$$
u=C_{1} u_{1}+C_{2} u_{2} \simeq e^{z}\left(\frac{z}{2}\right)^{1 / 5} \frac{1}{2 \sqrt{\pi}}\left(C_{2}+C_{1}\right),
$$

and, consequently, the asymptotic behavior of this approximate solution is given by formulae (56) with

$$
\mathcal{A}=\frac{1}{2^{6 / 5} \sqrt{\pi}}\left(C_{1}+C_{2}\right)<0
$$

and $\mathcal{B}>0$. At $t_{0} \gg 1$

$$
\mathcal{A} \approx-0.77 \frac{g^{2}}{16 \pi^{2}}, \quad \mathcal{B} \approx 3.24
$$


and

$$
\Delta_{r} \simeq \frac{0.308}{p_{e}^{2} \log ^{4 / 5} \frac{p_{e}^{2}}{M^{2}}}
$$

\section{Conclusions}

Our results demonstrate that two-particle approximation of the system of SchwingerDyson equations essentially differs in the asymptotic deep-Euclidean region of momenta in comparison with the MF approximation. Instead of physically unsatisfactory behavior with the Landau pole in the Euclidean region, which occurs for the MF approximation, or for the leading term of $1 / N$-expansion, the boson propagator in the two-particle approximation has self-consistent asymptotic behavior, which is similar to the asymptotically free behavior. Certainly, inclusion of the scalar self-action into consideration can notably vary the results in the asymptotic

region. From this point of view, the obtained results should be considered as the first step of investigation of the full model, which will include Yukawa interaction and the self-action of the scalar field.

\section{Aknowlegement}

Author is grateful to V A Petrov for useful discussion.

\section{References}

[1] Landau L D et al 1954 Dokl.Akad.Nauk Ser.Fiz. 95 1177;

Landau L D and Pomeranchuk I Ya 1955 Dokl.Akad.Nauk Ser.Fiz. 102489

[2] Abrikosov A A et al 1954 Dokl.Akad.Nauk Ser.Fiz. 97 793;

Landau L D et al 1956 Suppl.Nuovo Cim. 3 80;

Pomeranchuk I Ya et al 1956 Phys.Rev. 103784

[3] Callaway D 1988 Phys.Reports 167241

Kleefeld F 2006 J.Phys.A: Math.Gen. A39 L9

[4] Frohlich J 1982 Nucl.Phys.B 200281

[5] Weinberg S 1995 The Quantum Theory of Fields Vol. II (Cambridge Univ. Press)

[6] Suslov I M 2011 JETP 112274 [ ZhETF 139319 ]

[7] Rochev V E 2011 J.Phys.A: Math.Theor. A44 305403

[8] Dahmen H D and Jona-Lasinio G 1967 Nuovo Cim.A 52807 
[9] Tanabashi M et al 1989 Proc. Dynamical Symmetry Breaking (Nagoya) (ed. T Mute and K Yamawaki), Nagoya Univ.Press, pp. 28-36

Clague D E and Ross G G 1991 Nucl.Phys.B 36443

Bashir A and Diaz-Cruz J L 1999 J.Phys.G: Nucl.Part.Phys. G25 1797

[10] Bogolyubov N N and Shirkov D V 1980 Introduction to the Theory of Quantized Fields (Wiley, New York )

[11] Luke Y L 1969 The Special Functions and their Approximations Vol. 1 (Academic Press, New York) 